INPLASY

PROTOCOL

To cite: Lu et al. Chinese Herbal Medicine Combined with EGFR-TKI in Advanced Nonsmall Cell Lung Cancer with EGFR Sensitive Mutation: a systematic review and metaanalysis protocol. Inplasy protocol 2020110063. doi: 10.37766/inplasy2020.11.0063

Received: 16 November 2020

Published: 16 November 2020

Corresponding author:

Yan Lu

luyan7@163.com

Author Affiliation:

Yueyang hospital of integrated traditional Chinese and western medicine, Shanghai University of Traditional

Chinese Medicine

Support: National

Administration of TCM.

Review Stage at time of this submission: The review has not yet started.

Conflicts of interest:

None.

\section{Chinese Herbal Medicine Combined with EGFR-TKI in Advanced Non- small Cell Lung Cancer with EGFR Sensitive Mutation: a systematic review and meta-analysis protocol}

Lu, Y1; Sun, CB2; Liu, Y3; Xu, J4; Deng, HY5; Jiao, LJ6; Gong, YB7, $\mathrm{Xu}, \mathrm{L}^{8}$.

Review question / Objective: Whether the addition of Chinese herbal medicine to EGFR-TKI prolongs PFS/OS and reduces adverse effect compared with EGFR-TKI+placebo in advanced non-small cell lung cancer patients harboring sensitive EGFR mutation.

Condition being studied: Optimizing the effect of EGFR-TKI is essential to improve long-term survival and quality of life for advanced non-small cell lung cancer patients with sensitive EGFR mutation.

INPLASY registration number: This protocol was registered with the International Platform of Registered Systematic Review and Meta-Analysis Protocols (INPLASY) on 16 November 2020 and was last updated on 16 November 2020 (registration number INPLASY2020110063).

\section{INTRODUCTION}

Review question / Objective: Whether the addition of Chinese herbal medicine to EGFR-TKI prolongs PFS/OS and reduces adverse effect compared with EGFRTKI+placebo in advanced non-small cell lung cancer patients harboring sensitive EGFR mutation.

Rationale: From the perspective of traditional Chinese medicine, EGFR-TKI impairs the Yin-Yang balance of body and results in drug resistance and adverse 
effect. Thus, the addition of Chinese herbal medicine (CHM) may help maintaining the balance, delaying TKI resistance and reducing side effect related to EGFR-TKI.

Condition being studied: Optimizing the effect of EGFR-TKI is essential to improve long-term survival and quality of life for advanced non-small cell lung cancer patients with sensitive EGFR mutation.

\section{METHODS}

Search strategy: Search strategies will be created by a clinical librarian with expertise in systematic review searching. The MEDLINE strategy will be developed with input from the review team, then peer reviewed by a second librarian, not otherwise associated with the review. After the MEDLINE strategy is finalized, it will be adapted to the syntax and subject headings of the other databases. The International Clinical Trials Registry Platform Search Portal, ClinicalTrials. gov and Chinese Clinical Trial Registry will be searched for ongoing or recently completed trials, and PROSPERO will be searched for ongoing or recently completed systematic reviews. As relevant studies are identified, reviewers will check for additional relevant cited and citing articles. A bridging search will be conducted prior to publication of the review to ensure inclusion of any relevant studies published since September 2020.

Participant or population: 1) Pathologically or cytologically confirmed with stage IV NSCLC; 2) harboring sensitive EGFR mutation; 3) Eastern Cooperative Oncology Group (ECOG) performance status (PS) scores of 0,1 , or 2 ; 4) age $\geq 18$ years; 5) estimated life expectancy of at least 12 weeks; 6) no major organ dysfunction.

Intervention: Chinese herbal medicine combined with EGFR-TKI. Chinese herbal medicine can be administered orally, externally or intravenously.

Comparator: EGFR-TKI+ placebo.

Study designs to be included: RCT.
Eligibility criteria: (1) Types of Studies. Randomized controlled trial (RCT) published in English and Chinese. (2) Types of Participants as mentioned above. (3) Types of Interventions. The experimental group received Chinese herbal medicine + EGFR-TKI. The control group received EGFR-TKI + placebo. (4) Types of Outcomes. The study must have at least one of following outcomes: PFS, OS, 1,2year survival rate.

Information sources: We will search China BioMedical Literature (CBM), Chinese National Knowledge Infrastructure (CNKI), Cqvip Database, Wanfang Database, MEDLINE (PubMed), EMBASE (Ovid), Google Scholar, and Cochrane Library from inception to date. Our search strategy will be developed using Medical Subject Headings (MeSH) and text words related to herb, traditional Chinese medicine, complementary and alternative medicine, EGFR-TKI, non-small cell lung cancer and EGFR mutation. The electronic database search will be supplemented by searching the grey literature via trial registries, Chinese clinical trial registry, clinicaltrial.gov, Google Advanced Search and by contacting authors where insufficient data are available. To ensure literature saturation, we will scan the reference lists of included studies or relevant reviews identified.

Main outcome(s): The primary endpoint is PFS, which is measured with the date of the videography from a random assignment to the date of objective progression or death by the researcher.

Additional outcome(s): The secondary endpoints include a comparison of OS, ORR, DCR, 1 and 2 year survival rate, quality of life (QoL), and safety. QoL is evaluated with the Functional Assessment of Cancer TherapyšCLung (FACT-L) questionnaire.

Data management: Two independent reviewers will screen titles and abstracts. Any discrepancies will be resolved through discussion after completion of screening and third-party opinion will be sought. After 
attaining full texts of screened studies, eligible papers will be selected by two independent reviewers based on the inclusion/ exclusion criteria in accordance with the PRISMA recommendation. Discrepancies will be resolved with discussion at the end of this stage and where necessary, third reviewer's opinion will be sought. Full text of two to five of the included studies will be selected randomly to evaluate the pilot data extraction questionnaire. Any further information that is not considered in the pilot form will be added after being approved by the review team. Title, abstract and full text of the included studies will then be carefully read by two independent reviewers to extract the required data according to a predefined questionnaire. Any existing discrepancies in data extraction will be discussed and where necessary, a third reviewer will be involved. If the included studies were sufficiently homogenous, meta-analysis of the aggregated data will be conducted. Calibration exercises will be performed at each level of the process. The results will be presented following the PRISMA flow diagram.

Quality assessment / Risk of bias analysis: The Cochrane Risk Bias Assessment Tool 5.1.0 will be used to assess the design and methods of the included papers and assess the risk of bias as well as applicability. The methodological issues related to the quality of RCT were the generation of treatment allocation, concealment of treatment, blinding, the completeness of the resulting data, selective reporting of findings, and other potential risks of bias. Two reviewers will independently conduct the quality assessment. Any disagreement will be solved by discussion and where required, third party consultation will be sought. The presence of potential biases within the studies will be reported descriptively.

Strategy of data synthesis: Meta-analysis was performed using Rev Man $\mathbf{5 . 3}$ software. The chi-squared test for heterogeneity was performed at first, and heterogeneity was presented as significant when 12 was over $50 \%$ or $P<0.1$. The random-effect model was used for the meta-analysis if significant heterogeneity was present, and the fixed-effect model was used when the heterogeneity was not significant. The effectiveness on PFS were measured and presented with risk ratio (RR) and $95 \%$ corresponding confidential interval $(\mathrm{Cl})$. Description analysis was performed when quantitative data could not be pooled. Funnel plots were used to analyze potential publication bias, and sensitivity analysis was performed.

Subgroup analys is: Subgroup summarization and analysis of the effect of CHM on PFS based on sex (male vs. female), age ( $>65 v s<65$ ), ECOG PS (0 vs. 1 vs. 2), staging (IIla vs. IIIb vs. IV), smoking status (yes vs. no), EGFR mutation status (19 Del vs. 21L858R vs. other rare mutations), TKIs (gefitinib vs. erlotinib vs. icotinib) will be conducted.

Sensibility analysis: We will conduct sensitivity analyses to examine results robustness from main analyses after subgroup analysis. We will assess the effects of excluding studies with controls receiving more than TKIs and studies with a high risk of bias in the areas of randomization process, deviations from intended interventions, missing outcomes, measurement of outcome and selection of reported results.

Language: Chinese and English.

Country(ies) involved: China.

Keywords: non-small cell lung cancer, EGFR sensitive mutations, EGFR-TKI, Chinese herbal medicine, drug resistance.

\section{Contributions of each author:}

Author 1 - Yan Lu - literature search, evaluation and review; manuscript draft.

Author 2 - Chenbing Sun - literature search, evaluation and review; manuscript draft.

Author 3 - Yu Liu - provide third-party opinion on any discrapancies between author 1 and 2.

Author 4 - Ji Xu - Data management and statistic analysis.

Author 5 - Hongyong Deng - Data management and statistic analysis. 
Author 6 - Lijing Jiao - topic selection and methodology guide.

Author 7 - Yabin Gong - topic selection and methodology guide.

Author 8 - Ling Xu - topic selection and methodology guide. 